\title{
Fracture propagation stimulated by hydraulic injection under stress field
}

\author{
Kurama OKUBO $^{1}$, Hitoshi MIKADA ${ }^{1}$ and Junichi TAKEKAWA ${ }^{1}$ \\ ${ }^{1}$ Dept. of Civil and Earth Res. Eng., Kyoto University
}

\begin{abstract}
Hydraulic fracturing is a major scheme for improving the production of unconventional oil and gas as well as in horizontal wells. Created fractures increase the permeability of reservoir formations, which are usually tight and of low permeability, through a network of fractures in the reservoir. Laboratory experiments indicate that hydraulic fractures would propagate in the direction of the maximum principal stress around the fracture tip. This indicates that in-situ stress could play an important role in the behavior of hydraulic fracture propagation in the field scale. It is, however, difficult to observe the fracture propagation directly due to the depth of the reservoir layer ( $>2 \mathrm{~km}$ generally). There are two types of rock failure that are suggested to take place at the hydraulic fracturing, (i) tensile and (ii) shear fractures. In earthquake seismology, we know the latter is dominant in the generation of natural earthquakes. However, the ratio of tensile to shear fracture events induced by the fluid injection has not been well investigated yet due to the small magnitudes of failures. To tackle this problem, we adopted the extended finite element method (X-FEM) and added a new degree of freedom for the effects of the fluid inside fractures. It would bring an idea on how fracture propagates in a stable stress field no matter how the magnitude of each event becomes small. We developed a hydraulic fracturing simulation tool to explore the mechanism of fracture propagation triggered by the fluid injection. For the evaluation of the fracture propagation, we assumed a numerical simulation model in real scale and put external forces as an in-situ stress. We conducted two types of simulations, one homogeneous and the other inhomogeneous in the rock strength distribution. The homogeneous model showed that fractures propagate with both tensile and shear failures even if the injected fluid acts homogeneously outward at the fluid-solid interface. The inhomogeneous model showed that fractures no longer propagate simply in the direction to the maximum principal stress field. Our results indicate that both tensile and shear failures take place even in the homogeneous model probably due to the influence of stress field, and that the propagation of small fractures takes place randomly in the inhomogeneous model due to localized small-scale inhomogeneous stress field acting at the tip of fractures.
\end{abstract}

\section{INTRODUCTION}

The hydraulic fracturing plays an important role to develop unconventional resources as well as the horizontal well and AE monitoring. Fluid injection creates fracture network in the reservoir, which improve the permeability to enhance the production of the unconventional resources. It was suggested from laboratory experiments that the direction of the fracture propagation induced by the fluid injection turns to the direction of the maximum principal stress of the in-situ stress field (Zoback et al. 1977). It is assumed that the fracturing mechanism, that means the mode of fracturing (mode I; tensile, mode II; shear), would depend on the in-situ stress field. Therefore, it is required to consider the influence of the in-situ stress and estimate the stress field around the fracture tip to evaluate the direction of the fracture propagation and the fracturing mechanism.
However, the stress distribution around the fracture tip could be disturbed by the stress concentration at the fracture tip, the effect of pre-existing fractures and the inhomogeneities of the medium. The stress field around the fracture tip has never been observed directly due to the scale of the fracturing and the depth of the formation. The frequency of the mode I and mode II crack during hydraulic fracturing has been unknown so far.

Shimizu et al. (2011) simulated the fracture propagation around bore hole with distinct element method (DEM), which infered the direction of the fracture propagation and the fracturing mechanics. While these studies focused on the fracturing simulation in the experimental scale $(<$ several meters) around bore hole, it would be difficult to expand it to the realistic scale ( $>$ tens of meters) due to the computational cost. It is required to estimate the change of the stress distribution with the fracture propagation and reduce the computatinoal 


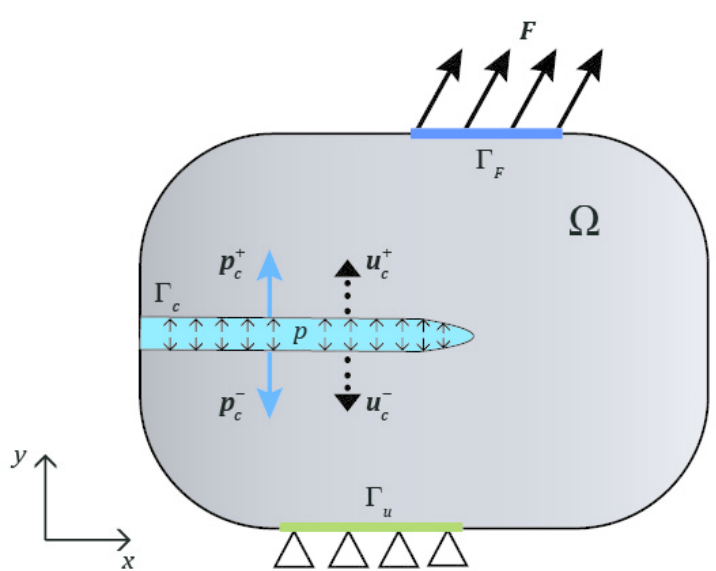

Figure 1 Schematic of the forces of hydraulic fracturing problem. Figure modified from Chen (2013).

cost to simulate the fracture propagation in a wide area to reveal the relationship between the in-situ stress and the mechanism of fracture propagation. We adopted the extended finite element method (XFEM), which was suggested by Belytschko and Black (1999). It reduces the computational cost of the calculation of the stress field with pre-existing fracture significantly by using additional degree of freedom (DOF) on the vertices of the element including the fracture. Chen (2013) extended this method to apply it to the hydraulic fracturing problem; the new DOFs concerning the pressure from the inner fluid of the fracture are added to the vertices. It succeeded to derive the static stress field around the pre-existing fracture with fluid injection. In this study, we used the optimized XFEM for the kinematic hydraulic fracturing problem and estimate stress intensity factors (SIFs) to evaluate the fracturing mechanism quantitatively. It was assumed that the fracture would propagate in mixed-mode even if the fluid were injected inside the fracture due to the in-situ stress. We would like to deduce the fracturing mechanism of hydraulic fracturing under in-situ stress field with the hydraulic fracturing simulation.

\section{METHODS}

In the XFEM, the secondary function is added into the conventional FEM to consider the effects of pre-existing fractures on the stress distribution, which is called enrichment. The function is defined as the enrichment function. The advantages of this scheme are following. a) Fractures are defined independent of the mesh, which could reduce the computational cost. b) It maintains the continuity of displacement at each node on the elements. It would

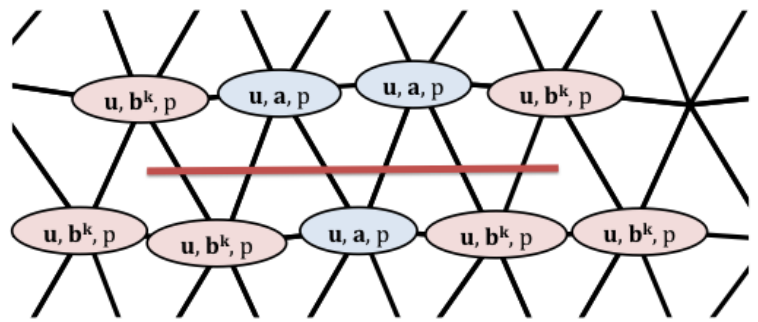

Figure 2 Additional DOFs. black line shows the elements and red line shows the pre-existing fracture.

bring stable stress field and fracture simulations.

The displacement approximation for six nodes triangle elements is defined as follows:

$$
\begin{aligned}
\boldsymbol{u}^{h}(\boldsymbol{x})= & \sum_{I=1}^{6} \boldsymbol{N}_{I}(\boldsymbol{x}) \boldsymbol{u}_{I}+ \\
& \sum_{I \in J} \boldsymbol{N}_{I}(\boldsymbol{x}) H(\boldsymbol{x}) \boldsymbol{a}_{I}+ \\
& \sum_{I \in C} \boldsymbol{N}_{I}(\boldsymbol{x}) \sum_{k=1}^{4} \gamma_{k}(\boldsymbol{x}) \boldsymbol{b}_{I}^{k} .
\end{aligned}
$$

$N_{I}$ is a shape function used by classical FEM. $C$ is the set of top nodes of finite elements, which includes crack tip, while $J$ is that which includes the intermediate part of the fracture. $\gamma_{k}$ is defined as

$$
\begin{aligned}
& \gamma_{1}=\sqrt{r} \cos \theta / 2, \gamma_{2}=\sqrt{r} \sin \theta / 2, \\
& \gamma_{3}=\sqrt{r} \sin \theta / 2 \sin \theta, \gamma_{4}=\sqrt{r} \cos \theta / 2 \sin \theta
\end{aligned}
$$

where $(r, \theta)$ are the local polar coordinates at the crack tip, and this expresses the stress concentration around the crack tip based on linear elastic fracture mechanics. $H$ is also defined as

$$
H(x)=\left\{\begin{array}{cc}
1 & x \in \Omega_{+} \\
0 & x \in \Gamma \\
-1 & x \in \Omega_{-}
\end{array}\right\} .
$$

This shows a jump function for taking account of the discontinuity of the bilateral region of the fracture, $\Omega_{+}$and $\Omega_{\text {. }}$.

To deal with the fluid-solid interaction analysis for the hydraulic fracturing problem, Chen (2013) proposed the additional DOFs for the effects of the inner fluid of the fracture. The equation of virtual work principle considering with the fluid pressure is shown as

$$
\begin{gathered}
\int_{\Omega}\left(\delta \boldsymbol{\varepsilon}^{T} \boldsymbol{\sigma}-\delta \boldsymbol{u}^{T} \mathbf{b}\right) d \Omega-\int_{\Gamma_{F}} \delta \boldsymbol{u}^{T} \mathbf{F} d \Gamma \\
-\left(\int_{\Gamma_{c}^{+}} \delta \boldsymbol{u}_{c}^{+T} \boldsymbol{p}_{c}^{+} d \Gamma+\int_{\Gamma_{c}^{-}} \delta \boldsymbol{u}_{c}^{-T} \boldsymbol{p}_{c}^{-} d \Gamma\right)=0,
\end{gathered}
$$


Table 1 Model parameter

\begin{tabular}{ll}
\hline node & 8665 \\
\hline element & 4262 \\
\hline $\mathrm{V}_{\mathrm{p} \text { background }}$ & $3000 \mathrm{~m} / \mathrm{s}$ \\
\hline Poisson's ratio & 0.3 \\
\hline
\end{tabular}

where $\sigma, \varepsilon$ are stress and strain respectively, $\boldsymbol{u}$ is

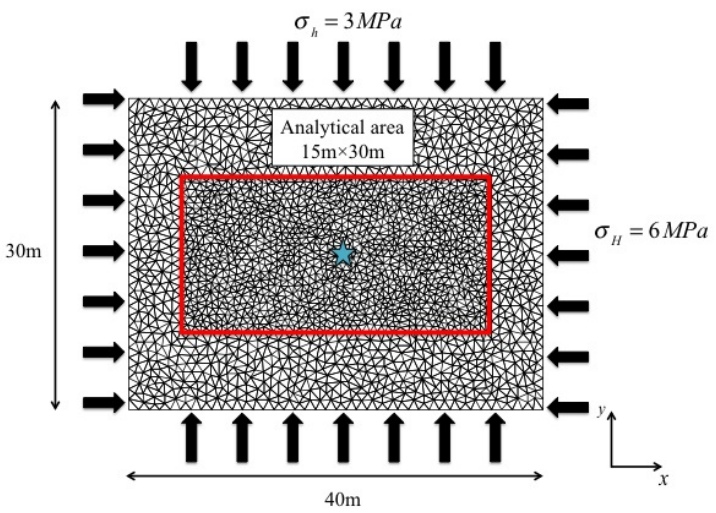

Figure 3 Simulation model. $\sigma_{H}$ and $\sigma_{h}$ are set as in-situ stress. Star shows the position of the injection well. The mesh sets finer around the injection well to improve the accuracy of calculation.

displacement of the body, $\mathbf{b}$ is body force, $\mathbf{F}$ is external force, $\boldsymbol{u}_{\boldsymbol{c}}$ and $\boldsymbol{p}_{\boldsymbol{c}}$ are the forces on the fracture surface shown in Figure 1. These notations are corresponded to Figure 1. The weak form of equation 4 is discretized with the approximation formula derived from the nodal value in the similar way as the conventional FEM. Figure 2 shows the way of the enrichment of new DOFs on each node.

\section{SIMULATION MODEL}

Figure 3 shows a two-dimensional simulation model used in this study. We used the triangle quadratic element. The model parameters are shown in table 1. To investigate the effects of the in-situ stress on the direction and mechanism of the fracturing propagation and the contribution of the local inhomogeneities of the geological formation to the fracturing mechanics, we conducted two simulations with the homogeneous and inhomogeneous models, and obtained the characteristics of the fracture propagation.

We modeled the inhomogeneous velocity structure based on the von Kármán autocorrelation function to reproduce the real inhomogeneities of the earth crust (Frankel and Clayton 1986).

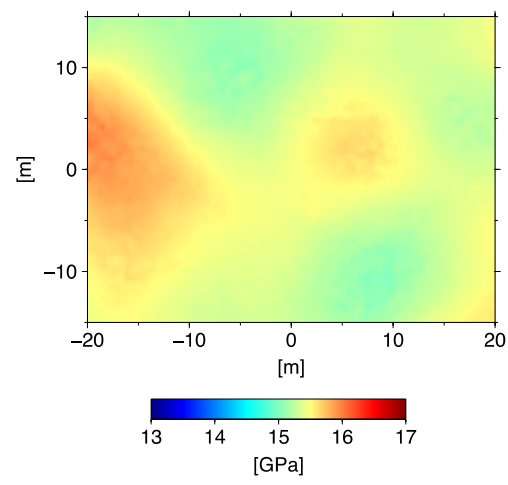

Figure 4 The inhomogeneous model of the Young's module. Contour shows the Young's module derived from the inhomogeneous velocity structure.

The von Kármán type autocorrelation function is

$$
N(r)=\frac{2^{1-v}}{\Gamma(v)}\left(\frac{r}{L}\right)^{v} K_{v}\left(\frac{r}{L}\right),
$$

where $\mathrm{L}$ is correlation length, $v$ is the Hurst number, which controls the complexity of the inhomogeneities, $K_{v}$ is modified Bessel function of order $v, \Gamma$ is the gamma function. We also apply the Gardner's relationship (Gardner et al. 1974) to derive the relationship between the density and $\mathrm{P}$ wave velocity. The Young's module $E$ is therefore derived from elastic wave mechanics that is

$$
E=\frac{(1+v)(1-2 v)}{1-v} \rho V_{p}^{2},
$$

where $v$ is Poisson's ratio, $\rho$ is density and $V_{p}$. is $\mathrm{P}$ wave velocity. Figure 4 shows the inhomogeneous model of Young's module using this method. We observed the disturbance of the stress field due to the local inhomogeneities and evaluated the effects of it on the fracturing mechanics.

\section{RESULTS AND DISCUSSIONS}

We first simulated fracture propagation in the homogeneous model. Figure 5 shows the simulation results of the homogeneous model. Figure 5(a) shows the snapshot of the stress distribution $(t=32$ min after injection). Figure 5(b) shows the trace of fracture propagation and the ratio of SIFs $\mathrm{KII} / \mathrm{KI}$ as an indicator of the fracturing mechanism. It is found that the coupled scheme makes it possible to estimate the stress distribution on the hydraulic fracturing problem and simulate the fracture propagation. In the homogeneous case, the fracture propagates in the direction of the maximum principal stress of the in-situ stress, which is in good agreement with the experimental results. In addition, 
it suggests that the ratio of the SIFs is

(a)

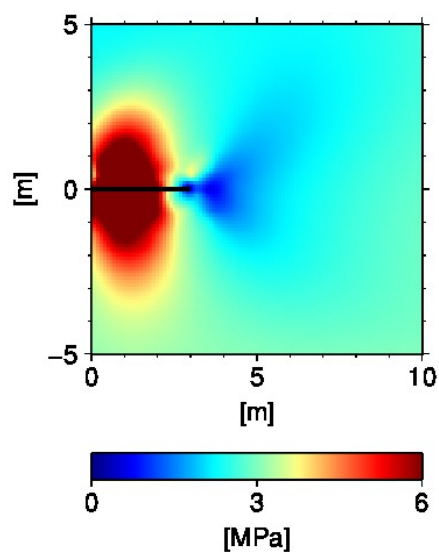

(b)

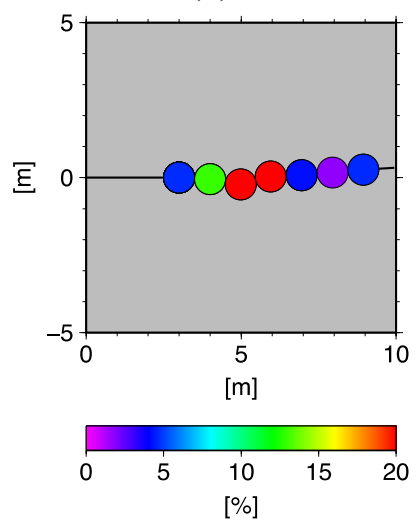

Figure 5 (a) Snapshot of the stress distribution around the fracture with homogeneous model. Black line shows fracture propagation and the contour shows the Mises stress. (b) The trace of the fracture propagation and the ratio of the SIFs KII/KI.

not zero at any crack point. It shows, therefore, that the fracture would propagate in mixed-mode even if the fluid is injected inside the fracture and presses in the tensile direction.

We then conducted the hydraulic fracturing simulation using the inhomogeneous model. Figure 6 shows the results of the fracture propagation and the ratio of the SIFs. The fracture propagates basically in the same direction of the maximum principal stress. However, the fracture does not propagate straight due to the disturbance of the stress field by the local inhomogeneities. The ratio of the SIFs KII/KI becomes higher than the homogeneous mode case, which means that the portion of the Mode II (shear component) increases in the inhomogeneous case. It suggests that the local inhomogeneities of the geological formation might disturb the stress field around the fracture, which could affect the fracturing mechanism.

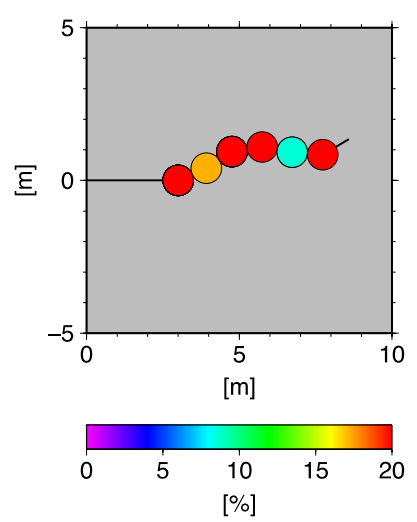

Figure 6 Fracture propagation and the SIFs ratio KII/KI with inhomogeneous model

\section{CONCLUSIONS}

We succeeded the hydraulic fracturing simulation with the enrichment of the new DOFs for the fluid pressure. The results showed that although fluid is injected inside the fracture, the fracture could propagates with mixed-mode crack, which has both Mode I (tensile) and Mode II (shear) components. It was also proposed that the local inhomogeneities of the geological formation could have an influence on the fracturing mechanism due to the change of the stress field around the fracture tip. These results could contribute to clarify the hydraulic fracturing mechanism.

\section{REFERENCES}

1) Zoback, M.D., Rummel, F., Jung, R. \& Raleigh, C.B., Laboratory hydraulic fracturing experiments in intact and pre-fractured rock. International Journal of Rock Mechanics and Mining Sciences \& Geomechanics Abstracts, 14, pp.49-58, 1977

2) Shimizu, H., Sumihiko, M. \& Tsuyoshi, I., The distinct element analysis for hydraulic fracturing in hard rock considering fluid viscosity and particle size distribution. International Journal of Rock Mechanics and Mining Sciences, 48(5), pp.712-27, 2011

3) Belytschko, T. \& Black, T., 1999, Elastic crack growth in finite elements with minimal remeshing. International journal for numerical methods in engineering, 45(5), pp.601-20.

4) Chen, Z., 2013, Implementation of the extended finite element method for hydraulic fracture problems. 13th International Conference on Fracture.

5) Frankel, A. \& Clayton, R.W., 1986, Finite difference simulations of seismic scattering: implications for the propagation of short-period seismic waves in the crust and models of crustal inhomogeneities. Journal of Geophysical Research, 91, pp.6465-89.

6) Gardner, G.H.F. \& Gardner, L.W., 1974, Formation velocity and density-the diagnostic basics for stratigraphic traps. Geophysics, 39, pp.770-80. 